\title{
Lactic-Acid Bacteria Supplement Fermented Dairy Products with Human Behavior-Modifying Neuroactive Compounds
}

\author{
Alexander V. Oleskin ${ }^{1, *}$, Olga G. Zhilenkova ${ }^{2}$, Boris A. Shenderov², \\ Adelaide M. Amerhanova ${ }^{2}$, Vladimir S. Kudrin ${ }^{3}$ and Peter M. Klodt ${ }^{3}$ \\ ${ }^{1}$ General Ecology Department, School of Biology, Lomonosov Moscow State University, Russia \\ ${ }^{2}$ Gabrichevsky Institute of Epidemiology \& Microbiology, Russian Academy of Medical Sciences, Russia \\ ${ }^{3}$ Zakusov Institute of Pharmacology, Russian Academy of Medical Sciences, Russia
}

\begin{abstract}
Using high performance liquid chromatography, we established that probiotic Lactobacillus strains (Lactobacillus helveticus 100ash, L. helveticus NK-1, L. casei $\mathrm{K}_{3} I_{1} \mathrm{I}_{24}$, and L. delbrueckii subsp. bulgaricus) grown on two milk-containing nutrient media produce important neuromediators such as biogenic amines, their precursors and deamination products, as well as neuroactive amino acids. The concentrations of biogenic amines (such as catecholamines and, with L. helveticus 100ash, also serotonin) equal or exceed those contained in the bloodstream of healthy adult humans, whereas those of most amino acids are comparatively low, except for gamma-aminobutyric acid (GABA). Of paramount importance is the fact that the bacterial cultures can release micromolar amounts of GABA and L3,4-dihydroxyphenylalanine (DOPA) into the milk-containing media. It is known that DOPA passes through the gut-blood and the blood-brain barrier and converts into major neurotransmitters (dopamine and norepinephrine) that influence important aspects of human behavior. The data obtained suggest that dairy products fermented by live lactobacillicontaining starters are potential sources of human behavior-modifying substances.
\end{abstract}

Keywords: Microbial endocrinology, fermented dairy products, probiotic lactobacilli, Lactobacillus helveticus, Lactobacillus casei, Lactobacillus delbrueckii subsp. bulgaricus, neuromediators, catecholamines, neuroactive amino acids, behavior modification, aggressiveness, dominance, biopolitics.

\section{INTRODUCTION}

One of the most dynamically developing interdisciplinary areas of research is microbial endocrinology [1-10]. Microbial endocrinology is related to both microbiology and medicine and deals with animal hormones, neuromediators, and their analogs/homologues that are involved in intercellular communication in pro- and eukaryotic uni- and multicellular organisms. This work specifically focuses on neuromediators, compounds that carry out or regulate impulse transmission between nervous cells across the synaptic cleft. Neuromediators, including biogenic amines, amino acids, and neuropeptides, perform essential functions in the human organism, especially in the central nervous system where they regulate the sleeping-waking rhythm, hunger and satiation, mood, pain sensitivity, attention, concentration, memory, and sexual desire. They influence decision-making and problem-solving as well as various aspects of social behavior-related personal features including aggressiveness, dominance, and submissiveness.

Importantly, neuromediators are produced by a wide variety of symbiotic and opportunistic bacteria and

*Address correspondence to this author at the General Ecology Department, School of Biology, Lomonosov Moscow State University, Russia;

Tel: +7-903-507-2258; E-mail: aoleskin@rambler.ru fungi $[2-4,5,9,11-17]$. Since some of these microorganisms are widely spread in nature and represent typical inhabitants of the human/animal organism, the available data suggest that they exert a considerable regulatory influence on the nervous and the endocrine system. For instance, our studies with a commensal strain, Escherichia coli MC4100, revealed that its cultural fluid contains tens of nanomols of catecholamines (norepinephrine and dopamine), as well as much higher - micromolar - amounts of the catecholamine precursor L-3,4-dihydroxyphenylalanine (DOPA). Bacillus cereus that spoils food and causes food poisoning releases norepinephrine and DOPA into the medium $[13,18,19]$. As for neuroactive (regulatory) amino acids, suffice it to mention that the normal human gastro-intestinal (GI) microbiota produces the inhibitory neuromediator gamma-aminobutyric acid (GABA); if, under pathological conditions, the microbiota fails to supply the organism with GABA, irritated bowel syndrome (IBS) and still more serious health problems may develop (reviewed, [20]).

Foodstuffs consumed by humans exert a strong influence on their physiological, psychological, and behavioral characteristics. Apart from dietetics, medicine, and biotechnology, nutritional issues are of relevance to modern biopolitics, an interdisciplinary field that focuses on the relationship between human biology and social behavior as related to political 
activities [6, 7, 21-23]. This relationship is partly due to the impact of neuroactive compounds of microbial origin that are present in food items and influence the functioning of the brain and, therefore, human behavioral traits. Modifying foodstuffs with neurochemicals-producing microorganisms enables us to subtly manipulate the behavior of human individuals and groups, including the level of aggressiveness and the status in dominance-submission hierarchies. This subject is receiving increasing attention in the literature $[4,6,8,17]$.

Of special importance in nutritional terms are dairy products. Recently, it has been revealed that dairy products contain various compounds that are capable of regulating the functioning of the human nervous system [6, 8, 13]. For example, we have established that commercially produced fermented dairy products such as yogurts with live starter cultures contain a number of neuroactive substances that were present at concentrations of $0.2-2 \mu \mathrm{M}$ (micromoles/l) [24]. They include biogenic amines such as norepinephrine and dopamine and also a number of amino acids including taurine and glycine. Of particular interest was the detection of high (micromolar) concentrations of DOPA in the tested yogurt samples. This catecholamine precursor can cross the gut-blood and the blood-brain barrier [25, 26]. In the brain, DOPA converts to dopamine, which can transform into norepinephrine. The catecholamines are involved in regulating locomotive activity and emotional responses to environmental stimuli; they influence such socially important human qualities as communicability, dominance, aggressiveness, sexuality, and others $[6$, $27,28]$.

These data give grounds for the suggestion that fermented dairy products are a potentially important source of several types of neuromediators (biogenic amines and amino acids) and their microbes produce concentrations that are sufficiently high to modify the pool of neurotransmitters controlling the neurological status of the human organism.

The goal of this work was to determine whether lactobacillus bacteria used as live starter cultures in industrially produced dairy products synthesize and release major brain neuromediators.

\section{MATERIALS AND METHODS}

The following strains of lactobacilli were used in this work: Lactobacillus helveticus 100ash, L. helveticus NK-1, L. casei $\mathrm{K}_{3} \|_{24}$, and $L$. delbrueckii subsp. bulgaricus. These cultures were obtained from the Russian State Collection of Human Normal Microbiota (Gabrichevsky Institute of Epidemiology \& Microbiology). Each of the strains of lactobacilli was grown on rehydrated $1 \%$ milk with a fat content of $0.5 \%$ or on the PHM medium containing pancreatic hydrolysate of milk $(500 \mathrm{ml})$, distilled water $(500 \mathrm{ml})$, sodium chloride $(4 \mathrm{~g})$, peptone $(2 \mathrm{~g})$, lactose $(10 \mathrm{~g})$, cysteine hydrochloride $(0.1 \mathrm{~g})$, and agar $(0.75 \mathrm{~g}) ; \mathrm{pH}$ 6.8-7.2. The milk medium was employed by us because it is industrially used for the microbial production of fermented dairy products including the yogurts tested in our previous studies [24]. The milk hydrolysate-based medium (PHM) contained amino acids and short polypeptide fragments resulting from caseine hydrolysis; additional amino acids and peptides were added by supplementing the PHM medium with peptone. This medium was used because it was enriched in "raw materials" from which living cells enzymatically synthesize biogenic amines, e.g., catecholamines and serotonin that are produced from the amino acids tyrosine and tryptophan, respectively. The other components contained in PHM were responsible for adjusting the osmolarity, redox potential, and acidity of this medium.

The bacteria were cultivated for 6 hours under microaerophilic conditions at $37^{\circ} \mathrm{C}$; the inoculum dose was $1.0 \times 10^{7} \mathrm{CFU} / \mathrm{ml}$. After 6 hours of cultivation, the medium $\mathrm{pH}$ value was $3.5-4.5$ and all tested strains formed a more or less dense clot; the cultivation was terminated by cooling the culture to $+4-6^{\circ} \mathrm{C}$. The CFU number of lactobacilli was determined by diluting culture samples in pre-reduced peptone saline containing $0,5 \mathrm{~g} / \mathrm{L}$ cysteine/ $\mathrm{HCl}(\mathrm{pH} \mathrm{6.3)}$ and plating them on MRS agar medium; the plates were incubated under microaerophilic conditions at $37^{\circ} \mathrm{C}$ for 48 hours. The CFU number before the termination of the fermentation process was $4.5-5.5 \times 10^{8} \mathrm{CFU} / \mathrm{ml}$.

The contents of biogenic amines including catecholamines (dopamine, DA, and norepinephrine, $\mathrm{NE}$ ) and serotonin $(5-\mathrm{HT})$, their precursors (L-3,4dihydroxyphenylalanine, DOPA, and 5hydroxytryptophan, 5-HTP), and metabolites (3,4dihydroxyphenylacetic acid, DOPAC, homovanillic acid, HVA, and 5-hydroxyindolylacetic acid, 5-HIAA) as well as neuroactive amino acids such as aspartic acid, glutamic acid, glycine, taurine, and $\gamma$-aminobutyric acid (GABA) were determined in the tested systems (sterile milk; PHM medium; or bacterial cultures grown on these media). In some experiments, bacterial biomass was separated by centrifugation $(8000 \mathrm{~g} ; 20 \mathrm{~min}$ ); the 
culture fluid supernatant (CF) was passed through a millipore filter (pore diameter $0.22 \mu$ ). The wet biomass sediment was sonicated (Braunsonic 1510, USA) at 22 $\mathrm{KHz}$; four to eight thirty-second pulses of sonication were carried out with one-minute spaces between them; the sonication was performed at $0^{\circ} \mathrm{C}$. The completeness of biomass disintegration was assessed with a light microscope at a magnification of 1000; the share of broken cells exceeded $90 \%$ in all samples.

The sonicated biomass (SM) was centrifuged (8000g; $20 \mathrm{~min}$ ), and the SM supernatant, the culture fluid supernatant, and the sterile media (milk and PHM) were used for high-performance liquid chromatography (HPLC) with an amperometric detection system. In a complex mixture of organic substances, the amperometric method provides for a high sensitivity of the detection system to extremely low concentrations of compounds of interest while its sensitivity threshold for the other components of the mixture is significantly higher [29].

The amperometric detection of HPLC-separated compounds is based upon measuring the electric current that is caused by oxidizing or reducing the tested compounds on the surface of the working electrode while a certain voltage is generated between the working electrode and the reference electrode [29]. The particular class of compounds that is detected in this work, biogenic amines and their derivatives, are oxidized when they come into contact with the working glass-carbon electrode; for instance, catecholamines, e.g., dopamine, convert into $p$-quinonimines. In addition to the compounds to be chromatographically separated and electrochemically detected, the tested solution contains an internal standard, 3,4-dihydroxybenzylamine (DHBA), at a fixed concentration of $0.5 \mu \mathrm{M} / \mathrm{l}$. The location and size of the DHBA peak on the chromatogram helped us calibrate the detection system.

An LC-304T chomatographer (BAS, West Lafayette, США) with a Rheodyne 7125 injector was employed; the volume of the loop used for applying samples was $20 \mu \mathrm{l}$. The tested biogenic amines were separated on a reverse-phase ReproSil-Pur column (ODS-3, 4x100 mm, $3 \mu$; Dr. Majsch GMBH, Elsico, Moscow). A PM-80 pump (BAS, USA) was used; the elution rate of the mobile phase was $1.0 \mathrm{ml} / \mathrm{min}$ at a pressure of $200 \mathrm{~atm}$. The mobile phase contained $0.1 \mathrm{M}$ citrate-phosphate buffer with $1.1 \mathrm{mM}$ octanesulfonic acid, $0.1 \mathrm{mM}$ EDTA, and $9 \%$ acetonitrile $(\mathrm{pH} 3.0)$. The measurements were carried out using an LC-4B electrochemical detector (BAS, USA) with a glass-carbon electrode $(+0.85 \mathrm{~V})$ against an $\mathrm{Ag} / \mathrm{AgCl}$ reference electrode. The samples were scanned with the Multichrome 1.5 (Ampersand) hardware-software system. All reagents used for the assay were analytical grade. The chromatographer was calibrated using a mixture of the tested biogenic amines; the concentrations of all these substances were $0.5 \mu \mathrm{M}$. The amine concentrations contained in the samples were calculated by the internal standard method that is based on determining the ratio between the peak area in the standard mixture and that in the samples, whereas the correct location and size of the peak of the internal standard (DHBA) demonstrated that the detection system was adequately calibrated [30].

The content of neuroactive acids (GABA, glycine, taurine, aspartic acid, and glutamic acid) in the experimental samples was determined by HPLC with a fluorimetric detection system using an LC-304T chromatographer (BAS, West Lafayette, USA) with a Phenomenex column $(\mathrm{C} 18,4 \times 150 \mathrm{~mm}, 4 \mu)$. A standard method modified by [31] was applied. The tested amino acids are weak chromophores, i.e., they do not emit or absorb UV light. Therefore, in order to reliably detect these amino acids, the samples were derivatized. For this purpose, we used o-phthalic aldehyde that forms a fluorescent complex with each of the tested amino acids. Derivatization was performed by supplementing $5 \mu \mathrm{l}$ of the dialysate with $10 \mu \mathrm{l}$ of 0.1 $\mathrm{M}$ borate buffer and $10 \mu \mathrm{l}$ of $o$-phthalic aldehyde-sulfate reagent in $0.1 \mathrm{M}$ borate buffer $(\mathrm{pH}$ 9.5). $0.01 \mu \mathrm{M}$ GABA, aspartic acid, glutamic acid, taurine, and glycine in $0.1 \mathrm{~N} \mathrm{HClO}_{4}$ were used as a standard calibration mixture. After $15 \mathrm{~min}$ of incubation at room temperature, $20 \mu \mathrm{l}$ of dialysate were applied to an Agilent Hypersil ODS column $(5 \mu \mathrm{M}, 4.6 \times 250)$. The substances separated were determined using an Agilent 1100 fluorescence detector (USA) with excitation and emission wavelengths of 230 and 392 $\mathrm{nm}$, respectively. The mobile phase for determining the neuroactive amino acids contained $0.06 \mathrm{M} \mathrm{NaH}_{2} \mathrm{PO}_{4} \mathrm{X}$ $\mathrm{H}_{2} \mathrm{O}, 3.2 \mathrm{mM} \mathrm{Na}_{2} \mathrm{HPO}_{4}, 0.025 \mathrm{mM}$ EDTA, and $1.24 \mathrm{mM}$ $\mathrm{CH}_{3} \mathrm{OH}$ (pH 5.6). The rate of the mobile phase was 1.5 $\mathrm{ml} / \mathrm{min}$.

The results obtained were statistically processed; the data given in the tables below are the means of 4-6 independent measurements.

\section{RESULTS}

Using HLPC, we established that both milk and the PHM medium contain a number of major neuroactive compounds; generally, their concentrations were higher 
in the PHM medium than in the milk. The tested starter cultures (L. helveticus 100ash, L. helveticus NK-1, L. casei $\mathrm{K}_{3} I_{24}$, and $L$. delbrueckii subsp. bulgaricus) significantly changed the neuromediators' concentrations in the two media. Predominantly, they increased these concentrations. Quantitative data on the concentrations of biogenic amines and amino acids are given in Tables 1-2 and Table 3, respectively.

L-3,4-Dihydroxyphenylalanine (DOPA): All tested cultures enriched both milk and PHM with DOPA, the catecholamine precursor (Table 1). This effect is particularly significant with $L$. casei $\mathrm{K}_{3} \mathrm{III}_{24}$ grown on milk and with $L$. helveticus NK-1 grown on the PHM medium. DOPA concentrations detected in total culture and culture fluid samples were similar. The biomass (intracellular content obtained by sonication) lacked DOPA, which suggests that DOPA is an extracellular compound in the lactobacillus cultures.

Dopamine (DA): The DA concentration slightly but statistically significantly increased (from 40 to $60-70$ $\mathrm{nM}$ ) if $L$. helveticus NK-1 and L. delbrueckii subsp. bulgaricus were cultivated in milk. The PHM medium initially (prior to inoculation) contained $\sim 6 \mu \mathrm{M}$ DA (Table 1), and the DA concentration decreased to zero in the presence of $L$. helveticus 100ash cells and to $0.5-2.5 \mu \mathrm{M}$ with the other tested cultures, i.e. the bacteria seemed to take up DA from the medium. Most DA was contained in the culture fluid; its intracellular content was zero in L. helveticus strain 100ash and $10-100 \mathrm{nM}$ in the other tested probiotic cultures.

Norepinephrine (NE): All tested bacterial cultures (except $L$. casei $\mathrm{K}_{3} \mathrm{III}_{24}$ ) caused a statistically significant increase in the NE concentration in both media. When cultivated on PHM, L. casei $\mathrm{K}_{3} \mathrm{II}_{24}$ decreased the NE concentration, compared to the control sample. Like $\mathrm{DA}, \mathrm{NE}$ was predominantly present in the culture fluid, and only a minor part of its pool appeared to bind to bacterial cell structures (see data on the intracellular content).

3,4-Dihydroxyphenylacetic Acid (DOPAC): In the presence of all tested bacterial strains, the concentration of DOPAC, the product of oxidative deamination of DA, increased $3-5$ fold in milk. Conversely, the cultivation of the lactobacillus strains in the PHM medium, which initially contained much DOPAC $(\sim 1 \mu \mathrm{M})$, resulted in decreasing the DOPAC concentration 1.5-10fold, both in the total culture samples and in the bacterial cell-free culture fluid.

Table 1: Catecholamines, their Precursor (DOPA), and Metabolites in Milk, Pancreatic Hydrolysate of Milk-Containing Medium (PHM), and Cultures of Lactobacilli

\begin{tabular}{|c|c|c|c|c|c|c|c|}
\hline \multirow{2}{*}{\multicolumn{3}{|c|}{ Strains, Substrates \& Fractions }} & \multicolumn{5}{|c|}{ Catecholamine Pathway } \\
\hline & & & DOPA & DA & NE & DOPAC & HVA \\
\hline \multirow{2}{*}{ No Bacteria } & \multicolumn{2}{|c|}{ Milk } & $0.05 \pm 0.03$ & $0.04 \pm 0.01$ & $0.02 \pm 0.01$ & $0.03 \pm 0.01$ & 0 \\
\hline & \multicolumn{2}{|c|}{ PHM } & $0.70 \pm 0.10$ & $6.17 \pm 0.50$ & $0.69 \pm 0.05$ & $1.20 \pm 0.25$ & 0 \\
\hline \multirow{4}{*}{ L. helveticus 100ash } & Milk & Total & $0.42 \pm 0.20$ & $0.04 \pm 0.20$ & $0.15 \pm 0.04$ & $0.15 \pm 0.03$ & 0 \\
\hline & \multirow{3}{*}{ PHM } & Total & $1.50 \pm 0.30$ & 0 & $0.09 \pm 0.02$ & $0.18 \pm 0.02$ & $0.08 \pm 0.02$ \\
\hline & & $\mathrm{CF}$ & $1.82 \pm 0.30$ & $0.05 \pm 0.01$ & 0 & $0.22 \pm 0.08$ & $0.10 \pm 0.03$ \\
\hline & & Cells & 0 & 0 & $0.10 \pm 0.02$ & 0 & 0 \\
\hline \multirow{4}{*}{ L. helveticus NK-1 } & Milk & Total & $0.08 \pm 0.02$ & $0.06 \pm 0.01$ & $0.12 \pm 0.03$ & $0.14 \pm 0.03$ & 0 \\
\hline & \multirow{3}{*}{ PHM } & Total & $5.37 \pm 1.00$ & $2.53 \pm 0.75$ & $2.32 \pm 0.50$ & $0.98 \pm 0.30$ & 0 \\
\hline & & CF & $3.71 \pm 1.00$ & $2.11 \pm 0.70$ & $1.94 \pm 0.45$ & $1.48 \pm 0.35$ & 0 \\
\hline & & Cells & 0 & $0.04 \pm 0.01$ & $0.10 \pm 0.02$ & 0 & 0 \\
\hline \multirow{4}{*}{ L. casei $\mathrm{K}_{3} \mathrm{II}_{24}$} & Milk & Total & $0.56 \pm 0.25$ & $0.03 \pm 0.01$ & $0.03 \pm 0.01$ & $0.15 \pm 0.04$ & 0 \\
\hline & \multirow{3}{*}{ PHM } & Total & $1.62 \pm 0.25$ & $0.59 \pm 0.10$ & $0.27 \pm 0.06$ & $1.04 \pm 0.20$ & 0 \\
\hline & & $\mathrm{CF}$ & $2.78 \pm 0.90$ & $0.22 \pm 0.04$ & $0.22 \pm 0.06$ & $1.08 \pm 0.25$ & 0 \\
\hline & & Cells & 0 & $0.10 \pm 0.02$ & $0.14 \pm 0.03$ & 0 & 0 \\
\hline \multirow{4}{*}{$\begin{array}{c}\text { L. delbrueckii subsp. } \\
\text { bulgaricus }\end{array}$} & Milk & Total & $0.11 \pm 0.03$ & $0.07 \pm 0.01$ & $0.16 \pm 0.05$ & $0.11 \pm 0.01$ & 0 \\
\hline & \multirow{3}{*}{ PHM } & Total & $4.00 \pm 1.00$ & $2.09 \pm 0.20$ & $3.47 \pm 0.55$ & $0.86 \pm 0.15$ & 0 \\
\hline & & $\mathrm{CF}$ & $3.40 \pm 0.80$ & $1.28 \pm 0.65$ & $2.17 \pm 0.25$ & $1.08 \pm 0.25$ & 0 \\
\hline & & Cells & 0 & $0.05 \pm 0.01$ & $0.09 \pm 0.02$ & 0 & 0 \\
\hline
\end{tabular}

All concentrations are expressed in micromoles per I $(\mu \mathrm{M})$. Designations: CF, culture fluid supernatant; Cells, intracellular content of the tested substances; DOPA L-3,4-dihydroxyphenylalanine; DA, dopamine; NE, norepinephrine (noradrenaline); DOPAC, dihydroxyphenylacetic acid; HVA, homovanillic acidt. 
Homovanillic Acid (HVA): HVA, another catecholamine metabolite, was only detected in the total sample of the fermented product and in the culture fluid of $L$. helveticus 100ash. It appears, therefore, to function as an extracellular compound.

5-Hydroxytryptophan (5-HTP): 5-HTP, the precursor of serotonin, was not detected in milk prior to inoculation. After cultivating the L. helveticus 100ash strain on milk, an atypically shaped peak appeared in the chromatogram close to the point where the 5-HTP peak should be located. These data on the presence of 5 -HTP in this sample should be regarded as dubious. Our results concerning the low concentrations of a 5HTP-similar substance determined in the PHM medium and in PHM-grown bacterial cultures have also been discarded by us as unreliable data.

Serotonin (5-Hydroxytryptamine, 5-HT): $0.4-0.5$ $\mu \mathrm{M} 5-\mathrm{HT}$ was detected in the total sample and the culture fluid of the $L$. helveticus 100 ash strain cultivated on PHM medium.

5-Hydroxyindolylacetic Acid (5-HIAA): Both media lacked this oxidized product of $5-\mathrm{HT}$. Tens of manomoles of 5-HIAA were detected in the biomass, the culture fluid, and the total samples of $L$. helveticus NK-1 or L. delbrueckii subsp. bulgaricus cultivated on these media. Since the cultures of these bacteria lacked 5-HT, it is difficult to account for the accumulation of its oxidized product. Presumably, the sensitivity threshold of the HPLC method was too high to detect $5-\mathrm{HT}$ in the cultures; alternatively, all 5-HT could rapidly convert to 5 -HIAA during the first hours of cultivation.

Aspartic Acid: This neuroactive amino acid was present in milk (prior to inoculation) at a concentration of $20-30 \mathrm{nM}$; the introduction of $L$. helveticus 100ash or $L$. helveticus NK-1 into the milk did not significantly change the concentration of this compound; whereas inoculating the two other strains resulted in completely removing aspartic acid from the medium. Aspartic acid was not detected in PHM whether with or without the tested lactobacilli.

Glutamic Acid was present in the milk medium at a concentration of $0.2 \mu \mathrm{M}$; all tested bacterial strains enriched milk in glutamic acid. The level of glutamic

Table 2: Serotonin (5-Hydroxytryptamine), its Precursor, and Metabolite in Milk, Pancreatic Hydrolysate of MilkContaining Medium (PHM), and Cultures of Lactobacilli

\begin{tabular}{|c|c|c|c|c|c|}
\hline \multirow{2}{*}{\multicolumn{3}{|c|}{ Strains, Substrates \& Fractions }} & \multicolumn{3}{|c|}{ Serotonin Pathway } \\
\hline & & & \multirow{2}{*}{$\begin{array}{c}\text { 5-HTP } \\
0\end{array}$} & \multirow{2}{*}{$\begin{array}{c}\text { 5-HT } \\
0\end{array}$} & \multirow{2}{*}{$\frac{\text { 5-HIAA }}{0}$} \\
\hline \multirow{2}{*}{ No Bacteria } & \multicolumn{2}{|c|}{ Milk } & & & \\
\hline & \multicolumn{2}{|c|}{$\mathrm{PHM}$} & 0 & 0 & 0 \\
\hline \multirow{4}{*}{ L. helveticus 100ash } & Milk & Total & $1.20 \pm 0.25^{\star}$ & 0 & 0 \\
\hline & PHM & Total & $0.49 \pm 0.05^{*}$ & $0.40 \pm 0.15$ & 0 \\
\hline & & CF & $0.47 \pm 0.05^{*}$ & $0.47 \pm 0.08$ & 0 \\
\hline & & Cells & 0 & 0 & $0.04 \pm 0.01$ \\
\hline \multirow{4}{*}{ L. helveticus NK-1 } & Milk & Total & 0 & 0 & 0 \\
\hline & PHM & Total & $0.77 \pm 0.30^{*}$ & 0 & $0.05 \pm 0.02$ \\
\hline & & $\mathrm{CF}$ & $0.79 \pm 0.35^{*}$ & 0 & $0.07 \pm 0.02$ \\
\hline & & Cells & 0 & 0 & $0.03+0.01$ \\
\hline \multirow{4}{*}{ L. casei $\mathrm{K}_{3} \mathrm{III}_{24}$} & Milk & Total & 0 & 0 & 0 \\
\hline & PHM & Total & $0.26 \pm 0.10^{*}$ & 0 & 0 \\
\hline & & $\mathrm{CF}$ & $0.17 \pm 0.05^{*}$ & 0 & 0 \\
\hline & & Cells & 0 & 0 & $0.06 \pm 0.01$ \\
\hline \multirow{4}{*}{ L. delbrueckii subsp. bulgaricus } & Milk & Total & 0 & 0 & 0 \\
\hline & PHM & Total & 0 & 0 & $0.08 \pm 0.02$ \\
\hline & & $\mathrm{CF}$ & 0 & 0 & $0.05 \pm 0.02$ \\
\hline & & Cells & 0 & 0 & $0.05 \pm 0.02$ \\
\hline
\end{tabular}

All concentrations are expressed in micromoles per I $(\mu \mathrm{M})$. Designations: $\mathrm{CF}$, culture fluid supernatant; Cells, intracellular content of the tested substances; 5 -HTP, 5-hydroxytryptophan; 5-HT, serotonin (5-hydroxytryptamine); 5-HIAA, 5-hydroxyindolylacetic acid.

Note: the data marked with an asterisk $\left({ }^{*}\right)$ are unreliable (see text). 
Table 3: Neuroactive Amino Acids in Milk, Pancreatic Hydrolysate of Milk-Containing Medium (PHM), and Cultures of Lactobacilli

\begin{tabular}{|c|c|c|c|c|c|c|c|}
\hline \multicolumn{3}{|c|}{ Strains, Media \& Fractions } & Aspartate & Glutamate & Glycine & Taurine & GABA \\
\hline \multirow{2}{*}{ No Bacteria } & \multicolumn{2}{|c|}{ Milk } & $0.02 \pm 0.01$ & $0.20 \pm 0.03$ & $0.13 \pm 0.02$ & $0.13 \pm 0.02$ & $0.02 \pm 0.01$ \\
\hline & \multicolumn{2}{|c|}{$\mathrm{PHM}$} & 0 & $0.09 \pm 0.02$ & $0.21 \pm 0.02$ & $0.88 \pm 0.03$ & $0.92 \pm 0.05$ \\
\hline \multirow{4}{*}{$\begin{array}{l}\text { L. helveticus } \\
\text { 100ash }\end{array}$} & Milk & Total & $0.03 \pm 0.01$ & $0.52 \pm 0.02$ & $0.31 \pm 0.10$ & $0.39 \pm 0.03$ & $0.24 \pm 0.02$ \\
\hline & \multirow{3}{*}{$\mathrm{PHM}$} & Total & 0 & $0.15 \pm 0.03$ & $0.28 \pm 0.03$ & $0.94 \pm 0.10$ & $0.63 \pm 0.10$ \\
\hline & & $\mathrm{CF}$ & 0 & $0.15 \pm 0.03$ & $0.27 \pm 0.03$ & $1.03 \pm 0.10$ & $0.88 \pm 0.04$ \\
\hline & & Cells & 0 & $0.04 \pm 0.01$ & $0.04 \pm 0.01$ & 0 & 0 \\
\hline \multirow{4}{*}{ L. helveticus NK-1 } & Milk & Total & $0.03 \pm 0.01$ & $0.59 \pm 0.04$ & $0.29 \pm 0.10$ & $0.42 \pm 0.03$ & $0.16 \pm 0.02$ \\
\hline & \multirow{3}{*}{ PHM } & Total & 0 & $0.18 \pm 0.04$ & $0.30 \pm 0.06$ & $0.93 \pm 0.10$ & $0.82 \pm 0.05$ \\
\hline & & $\mathrm{CF}$ & 0 & $0.16 \pm 0.02$ & $0.27 \pm 0.02$ & $1.06 \pm 0.06$ & $0.80 \pm 0.04$ \\
\hline & & Cells & 0 & $0.08 \pm 0.02$ & $0.06 \pm 0.01$ & 0 & 0 \\
\hline \multirow{4}{*}{ L. casei $\mathrm{K}_{3} \| I_{24}$} & Milk & Total & 0 & $0.62 \pm 0.10$ & $0.24 \pm 0.06$ & $0.47 \pm 0.05$ & $0.26 \pm 0.02$ \\
\hline & \multirow{3}{*}{$\mathrm{PHM}$} & Total & 0 & $0.14 \pm 0.02$ & $0.26 \pm 0.06$ & $0.97 \pm 0.10$ & $0.84 \pm 0.03$ \\
\hline & & $\mathrm{CF}$ & 0 & $0.15 \pm 0.03$ & $0.22 \pm 0.05$ & $0.95 \pm 0.10$ & $0.80 \pm 0.10$ \\
\hline & & Cells & 0 & $0.08 \pm 0.02$ & 0 & $0.03 \pm 0.01$ & 0 \\
\hline \multirow{4}{*}{$\begin{array}{c}\text { L. delbrueckii } \\
\text { subsp. bulgaricus }\end{array}$} & Milk & Total & 0 & $0.53 \pm 0.10$ & $0.28 \pm 0.10$ & $0.38 \pm 0.07$ & $0.32 \pm 0.02$ \\
\hline & \multirow{3}{*}{ PHM } & Total & 0 & $0.17 \pm 0.02$ & $0.25 \pm 0.06$ & $0.93 \pm 0.08$ & $0.90 \pm 0.04$ \\
\hline & & $\mathrm{CF}$ & 0 & $0.16 \pm 0.02$ & $0.24 \pm 0.06$ & $0.95 \pm 0.10$ & $0.85 \pm 0.03$ \\
\hline & & Cells & 0 & $0.04 \pm 0.01$ & 0 & 0 & 0 \\
\hline
\end{tabular}

All concentrations are expressed in micromoles per I $(\mu \mathrm{M})$. Designations: CF, culture fluid supernatant; Cells, intracellular content of the tested substances; GABA $\gamma$-aminobutyric acid.

acid in the fermented milk reached 0.5-0.6 $\mu \mathrm{M}$. Approximately $0.1 \mu \mathrm{M}$ glutamic acid was detected in $\mathrm{PHM}$; the glutamic acid concentration increased $\sim 1.5$ fold during the growth of all strains of lactobacilli. Glutamic acid wss predominantly present in the culture fluid and apparently was bound to cells to an insignificant extent.

Glycine was present in milk and PHM at concentrations of $\sim 0.1$ and $\sim 0.2 \mu \mathrm{M}$, respectively. Milk was enriched in glycine to a larger extent than PHM during the growth of all tested bacterial strains. Most glycine was contained in the cultural fluid.

Taurine was detected in milk at a concentration of about $0.1 \mu \mathrm{M}$; all tested bacteria increased its concentration to $0.4-0.5 \mu \mathrm{M}$. Approximately $1 \mu \mathrm{M}$ taurine was present in PHM initially; its concentration did not significantly change during the growth of all tested cultures. Taurine was mainly contained in the culture fluid.

$\gamma$-Aminobutyric Acid (GABA) was present in milk at low concentrations $(\sim 20 \mathrm{nM})$; all tested cultures significantly enriched this medium in GABA; the maximum increase was attained with $L$. delbrueckii subsp. bulgaricus that brought the GABA concentration up to $0.3 \mu \mathrm{M}$. GABA was contained in the culture fluid and, therefore, not bound to microbial cells.

\section{DISCUSSION}

Our research provides evidence that fermented milk products containing live lactobacilli are considerably enriched in neuroactive compounds including catecholamines (dopamine and norepinephrine), their precursor (DOPA), and their oxidation products; serotonin only appears in the culture of $L$. helveticus 100 ash cultivated on the PHM medium.

Interestingly, the concentrations of neuroactive compounds produced by the tested starter cultures are similar to those that are characteristic of the human bloodstream or even exceed these concentrations. Human blood contains [25, 27, 32], on average, $\sim 10$ nM DOPA, 0.1-0.5 nM DA (DA in the free form; human blood also contains $\sim 20-30 \mathrm{nM}$ sulfoconjugated DA [32]) and about $1 \mathrm{nM} \mathrm{NE}$. The fermented milk products inoculated by all tested bacteria contained $\sim 0.5-5 \mu \mathrm{M}$ DOPA, with the NK-1 strain on PHM yielding the 
maximum amount of DOPA, $5.37 \pm 1.00 \mu \mathrm{M}$ (Table 1). $L$. helveticus NK-1 and L. delbrueckii subsp. bulgaricus increased the DA concentration from 0.04 to $0.06 \mu \mathrm{M}$, i.e., by $20-30 \mathrm{nM}$, and most tested strains added some $0.1 \mu \mathrm{M} \mathrm{NE}$ to the milk medium (see Table 1). The blood concentration of the dopamine metabolite DOPAC mostly is within the $10-20 \mathrm{nM}$ range [32]; the DOPAC concentration with the tested microbial cultures was approximately within the same range (with NK-1 and L. bulgaricus on PHM) or at a somewhat lower level. Human blood also normally contains 0.5 $1.5 \mu \mathrm{M} 5-\mathrm{HT}$ [33], and the 0.4-0.5 $\mu \mathrm{M} 5-\mathrm{HT}$ level we detected in the culture fluid of $L$. helveticus 100ash is close to its minimum amount in the bloodstream.

Importantly, microbial cultures accumulate DOPA in milk and the pancreatic milk hydrolysate medium. As mentioned in the Introduction, the catecholamine precursor can penetrate into the brain tissue $[25,26]$ where it transforms into catecholamines that perform essential functions as major brain neuromediators. Normalizing the brain levels of DOPA and the neurochemicals produced from it helps overcome depression, adynamia, and other negative consequences of stress $[6,27,28]$.

Of some interest is the accumulation of neuroactive amino acids (glutamic acid, glycine, taurine, and GABA) during the cultivation of starter cultures of lactobacilli in the milk-containing media. The tested bacteria produce submicromolar (over $0.1 \mu \mathrm{M}$ ) amounts of neuroactive amino acids (except for aspartic acid). However, human blood plasma normally contains tens to hundreds of micromoles of most amino acids [26]. Therefore, the microbially produced concentrations of them seem insignificant in neurophysiological terms.

Nevertheless, GABA is contained in the human organism in amounts (about $0.6 \mu \mathrm{M}$ in blood plasma and $0.3 \mu \mathrm{M}$ in cerebrospinal fluid [34]) that are close to those released by the lactobacilli. For instance, our strain of $L$. delbrueckii subsp.bulgaricus produced $0.32 \pm 0.02 \mu \mathrm{M}$ on the milk medium. It is known that GABA, an inhibitory amino acid, produces a relaxing and pacifying effect; it is used as a tranquilizer which causes no addiction. GABA improves concentration and memory and normalizes the sleeping rhythm. It promotes the restoration of the locomotion- and speech-controlling neural networks after an injury and stimulates the metabolic processes in the brain that are involved in utilizing glucose and removing toxic metabolites [6, 31, 35].
The results obtained indicate that fermented dairy products and their starter cultures, including those used as probiotics, can serve as sources of behaviormodifying neurochemicals having the potential to modify the operation of the human brain and, therefore, to significantly influence human behavior. This conclusion is in line with the suggestion of a number of microbiologists and clinicians that gut symbiotic microorganisms-based probiotics can be used to modify the neurological and mental state of humans, e.g., for the purpose of decreasing the risk of neurological diseases $[3,4,15-17,36,37]$. Our data also demonstrate that functionally specialized food items can be developed that produce significant effects on the human brain, the mind, and social behavior. Biopolitical measures should be taken on the global scale that would enable us to efficiently monitor the concentrations of neuroactive compounds in dairy products and other food items.

\section{REFERENCES}

[1] Lyte M. The role of microbial endocrinology in infectious disease. J Endocrinol 1993; 137: 343-5. http://dx.doi.org/10.1677/joe.0.1370343

[2] Lyte M. The microbial organ in the gut as a driver of homeostasis and disease. Med Hypotheses 2010; 74: 634-8. http://dx.doi.org/10.1016/i.mehy.2009.10.025

[3] Lyte M. Probiotics function mechanistically as delivery vehicles for neuroactive compounds: microbial endocrinology in the design and use of probiotics. Bioessays 2011; 33: 57481

http://dx.doi.org/10.1002/bies.201100024

[4] Lyte M. Microbial endocrinology in the microbe-gut-brain axis: how bacterial production and utilization of neurochemicals influence behavior. PLoS Pathog 2013; 9(11): e1003726. http://dx.doi.org/10.1371/journal.ppat.1003726

[5] Shenderov BA. Impact of the microbial factor on the formation of the pool of free histamine and other amines in the host organism in relation to the development of allergic responses. In: Medical Microbial Ecology and Functional Nutrition. Vol. 2. The Social and Ecological Consequences of an Imbalance in Animal/Human Microbial Ecology. Moscow: Grant 1998; pp. 227-48.

[6] Oleskin AV. Biopolitics. The political potential of the life sciences. Hauppauge (New York): Nova Science Publishers 2012.

[7] Oleskin AV. Network structures in biological systems and in human society. Hauppauge (New York): Nova Science Publishers 2014.

[8] Oleskin AV, Shenderov BA. Biopolitical approach to rehabilitation: the potential impact of microbial neurochemistry. A review. Regen Med J 2013; 1: 60-7.

[9] Roshchina VV. Evolutionary considerations of neurotransmitters in microbial, plant, and animal cells. In: Lyte M, Freestone PP, editors. Microbial endocrinology: interkingdom signaling in infectious disease and health. New York: Springer Science + Business Media 2010; pp. 17-52.

[10] Shenderov BA. Innovative products and driver ingredients in the dairy market. Dairy Industry 2013; 6: 62-6.

[11] Voropaeva EA. Antibiotic resistance and histamine production by the bacteria isolated from the oro-pharynx of 
children with bronchial asthma. Antibiotics Chemotherapy 2002; 3: 19-23.

[12] Forsythe $\mathrm{P}$, Sudo $\mathrm{N}$, Dinan T, Taylor VH, Bienenstock J. Mood and gut feelings. Brain Behav Immun 2010; 24: 9-16. http://dx.doi.org/10.1016/j.bbi.2009.05.058

[13] Oleskin AV, Shishov VI, Malikina KD. Symbiotic biofilms and brain neurochemistry. Hauppauge (New York): Nova Science Publishers 2010.

[14] Barrett E, Ross RP, O'Toole PW, Fitzgerald GF, Stanton C. Gamma-aminobutyric acid production by culturable bacteria from the human intestine. J Appl Microbiol 2012; 113: 411-7. http://dx.doi.org/10.1111/j.1365-2672.2012.05344.x

[15] Cryan JF, Dinan TG. Mind-altering microorganisms: the impact of the gut microbiota on brain and behavior. Nature Rev Neurosci 2012; 13(10): 701-12 http://dx.doi.org/10.1038/nrn3346

[16] Saulnier DM, Ringel Y, Heyman MB, et al. The intestinal microbiome, probiotics and prebiotics in neurogastroenterology. Gut Microbes 2013; 4: 17-27. http://dx.doi.org/10.4161/gmic.22973

[17] Foster JA. Gut feelings: Bacteria and the brain. Cerebrum [serial on the Internet]. 2013 July 1 [cited 2014 July 30]. Available from: http://www.ncbi.nlm.nih.gov/pmc/articles/ PMC3788166

[18] Shishov VA, Kirovskaya TA, Kudrin VS, Oleskin AV. Amine neuromediators, their precursors, and oxidation products in the culture of Escherichia coli K-12. Appl Biochem Microbiol 2009; 45(5): 494-7. http://dx.doi.org/10.1134/S0003683809050068

[19] Shishov VA. Biogenic amines and their dynamics during the growth of microbial cultures. Cand. Sci. [Biol.] Dissertation. Moscow: Biology Faculty, Moscow State University 2010.

[20] Bai AP, Ouyang Q. Probiotics and inflammatory bowel diseases. Postgraduate Med J 2006; 82: 376-82. http://dx.doi.org/10.1136/pgmj.2005.040899

[21] Masters RD. Biology and politics: linking nature and nurture. Ann Rev Pol Sci 2001; 4: 345-69. http://dx.doi.org/10.1146/annurev.polisci.4.1.345

[22] Blank RH, Hines SM. Biology and political science. London: Routledge 2001.

[23] Somit A, Peterson SA. Introduction. In: S. A. Peterson SA, A. Somit A, editors. Research in Biopolitics. Vol.9. Biology and politics. The cutting edge. UK, North America, Japan, etc.: Emerald Group Publ. Ltd. 2011; pp. 3-10.

[24] Zhilenkova OG, Shenderov BA, Klodt PM, Kudrin VS, Oleskin AV. Dairy products as potential sources of compounds that modify their consumers' behavior. Dairy Industry 2013; 10: 16-9.

[25] Dubynin VA, Kamensky AA, Sapin MP, Sivoglazov VN. Regulatory systems of the human organism. Moscow: Drofa 2003.

[26] MedLine Plus. Plasma amino acids. Rockville Pike Bethesda, MD: U.S. Department of Health and Human Services National Institutes of Health [updated 2014 july 9; cited 2014 July 30]. Available from: http://www.nlm.nih.gov/ medlineplus/ency/article/003361.htm

[27] Orlov RS, Nozdrachev AD. Normal physiology. Moscow: GEOTAR-Media 2009.

[28] Eisenhofer G, Kopin IJ, Goldstein DC. Catecholamine metabolism: a contemporary view with implications for physiology and medicine. Pharmacol Rev 2004; 56(3): 33149. http://dx.doi.org/10.1124/pr.56.3.1

[29] Yashin AY, Yashin YI. Measuring options with a TsvetYauza liquid chromtographer equipped with electrochemical detectors. Ros Khim J 2002; 4: 1090-15.

[30] Kudrin VS, Klodt PM, Narkevich VB, et al. The behavioral and neurochemical aspects of the antidepressive action of GSB-106, a dipeptide fragment of brain neurotrophic factor BDNF. Exp Clin Pharmacol 2012; 75: 41-5.

[31] Khoroshilova IE, Panov PB. Clinical Nutritiology. Moscow: Nauka 2006.

[32] Eldrup E. Significance and origin of DOPA, DOPAC and dopamine-sulfate in plasma, tissue and cerebrospinal fluid. Dan Med Bull 2004; 31: 34-62.

[33] McPherson RA, Pincus MR, editors. Henry's Clinical Diagnosis and Management by Laboratory Methods. Philadelphia: Elsevier Saunders 2011.

[34] Abbott RJ, Pye IF, Nahorski SR. CSF and plasma GABA levels in Parkinson's disease. J Neurol Neurosurg Psychiatry 1982; 45: 253-56. http://dx.doi.org/10.1136/jnnp.45.3.253

[35] Potter B, Orfali S. Brain boosters. Foods and drugs that make you smarter. Berkeley (CA): Ronin Publishing 1993.

[36] Douglas-Escobar V, Elliott E, Neu J. Effect of intestinal microbial ecology on the developing brain. JAMA Pediatr 2013; 167(4): 374-79. http://dx.doi.org/10.1001/jamapediatrics.2013.497

[37] Hsiao EY, McDride AW, Hsien S, et al. Microbiota modulates behavioral and physiological abnormalities associated with neurodevelopmental disorders. Cell 2013; 155: 1451-63. http://dx.doi.org/10.1016/j.cell.2013.11.024 